\title{
Intervening on the Side Effects of Hormone-Dependent Cancer Treatment: The Role of Strength Training
}

\author{
Erik D. Hanson and Ben F. Hurley \\ Department of Kinesiology, School of Public Health, University of Maryland, College Park, MD 20742, USA \\ Correspondence should be addressed to Ben F. Hurley, benhur@umd.edu
}

Received 1 October 2010; Accepted 15 December 2010

Academic Editor: Iris Reuter

Copyright (C) 2011 E. D. Hanson and B. F. Hurley. This is an open access article distributed under the Creative Commons Attribution License, which permits unrestricted use, distribution, and reproduction in any medium, provided the original work is properly cited.

\begin{abstract}
While prostate and breast cancers are both highly prevalent and treatable using hormone suppression therapy, a constellation of side effects ensue, which mimic typical aging effects but at an accelerated pace. Because strength training is considered to be an intervention of choice for addressing the musculoskeletal and metabolic consequences of normal aging in older adults, it may be an effective intervention to attenuate or reverse the side effects of hormone-dependent cancer treatment. This paper provides an overview of the independent effects of strength training on common musculoskeletal and metabolic side effects of hormonedependent therapy used for prostate and breast cancers. Strength training appears to be an effective complementary therapy for some of the adverse effects of prostate and breast treatment. Future research needs to address potential mechanisms to explain recent findings and to explore the role of strength training in addressing specific risk factors resulting from cancer treatment.
\end{abstract}

\section{Introduction}

Cancer is the leading cause of death worldwide, accounting for 7.4 million deaths ( $\sim 13 \%$ of all fatalities) in 2004 and is projected to rise to more than 12 million deaths by the year 2030 [1]. Prostate cancer (PCa) in men (28\% of all cancer sites) and breast cancer $(\mathrm{BCa})$ in women $(28 \%)$ are the most prevalent types of cancer in the US [2]. They are the second leading causes of cancer-related deaths for men and women in the US, respectively [2], while BCa is the leading cause of cancer death in women across the globe [1].

The prevalence of all cancers increases with age [2], with those $\geq 65$ years of age accounting for $>60 \%$ of all cancers [3]. Because this age group is also more likely to have other comorbid conditions, such as osteoporosis, arthritis, and cardiovascular disease than younger age groups [3], treatment options become more complicated. Moreover, these patients tend to avoid physical activity, leading to some of the same adverse consequences as those previously experienced by cardiac patients who failed to engage in physical activity [4]. As is the case for cardiac patients, this course of action can result in losses of functional capacity and quality of life (QoL) measures for cancer patients. In contrast, regular exercise can reduce cancer-related fatigue and other adverse symptoms of the disease, as well as substantially improve the QoL in cancer patients $[4,5]$. Because five-year survival rates in localized $\mathrm{PCa}$ and $\mathrm{BCa}$ remain high [2], QoL related issues are of great concern. Although regular exercise has long been recommended for cancer patients, investigators have only recently focused on some of the specific musculoskeletal and metabolic adverse consequences ( side effects) of cancer treatment, particularly those of hormone ablation medications.

There appears to be an interesting parallel between the many side effects of both $\mathrm{PCa}$ and $\mathrm{BCa}$ treatment and the changes that occur with typical aging [6-9], but at an accelerated pace. Given the role of strength training (ST) as a countermeasure for the age-related changes in body composition and physical function [10], this review will focus on the effects of ST on the side effects elicited from medications used in the two most common hormonedependent cancers, that is, PCa and BCa. It will not include combined exercise training programs (e.g., those using both aerobic and resistance exercise) unless combined training 
programs are the only source of information available on the side effect being discussed.

\section{Treatments for PCa and BCa}

There is a wide variety of treatment options for both PCa and $\mathrm{BCa}$, depending on a patient's medical history, diagnosis, age, and current state of health. Although primary treatment for PCa may include radical prostatectomy, radiation, and other therapies, a common adjuvant treatment is the suppression of endogenous testosterone through the use of hormonal agents [11]. Likewise, while surgery is the primary therapy for BCa treatment, hormone suppression therapy serves as an important adjuvant therapy [12]. Chemotherapy is also used as a systemic treatment for BCa, either before surgery (neoadjuvant) or after (adjuvant) as needed. However, a common consequence of chemotherapy is ovarian failure $[13,14]$ and the onset of premature menopause.

Because most PCas are androgen dependent, androgen deprivation therapy (ADT) is a commonly used treatment for $\mathrm{PCa}$ [11] and results in what is sometimes referred to as a chemical castration. Although this treatment slows the growth of existing tumors, thereby potentially saving lives, the associated suppression of testosterone also leads to numerous adverse side effects, including an increase in the number of comorbidities, which ultimately have a negative impact on QoL.

Analogous to the treatment of PCa, hormonal therapy for the treatment of $\mathrm{BCa}$ is to inhibit the effects of estrogen. This can be accomplished using selective estrogenreceptor modulators, such as tamoxifen [15], or aromatase inhibitors, another example of an antiestrogen treatment [12]. Both treatments reduce growth in estrogen-responsive tumors. Historically, tamoxifen has been a mainstay in $\mathrm{BCa}$ treatment and can be administered to both premenopausal and postmenopausal women [15] while aromatase inhibitors are typically reserved for postmenopausal women [12].

\section{Side Effects of PCa and BCa Therapy and Strength Training}

\subsection{Does Hormone Therapy Mimic Aging Effects?}

Sex hormone levels decline gradually with age in men and, abruptly, with menopause in women. Previously, the cessation of endogenous production of androgens and estrogens during $\mathrm{PCa}$ and $\mathrm{BCa}$ treatment has been equated with increases in hormone-dependent age-related changes $[6,9]$. While it is evident that treatment of hormone-dependent cancers via ablation or modulation of the sex hormones is effective in shrinking tumor masses and extending longterm survival rates, such treatments also bring about declines in muscle mass [16-20], strength [21, 22], and bone mineral density (BMD) $[17,22-27]$ and increases in fat mass $[16,17,19-21,28,29]$, insulin resistance [30-33], and fatigue $[17,34-36]$. Collectively, these effects negatively influence physical function [18, 22, 37-39] and QoL $[21,34,36,40,41]$.
ST has been advocated as the intervention of choice for addressing many age-related declines $[7,8]$. In this regard, many researchers have demonstrated that ST can reverse age-associated losses in muscle mass $[42,43]$, strength [44], muscle power [42, 45], bone loss [46], insulin sensitivity $[47,48]$, and increases in regional fat deposition [49], as well as the deterioration of muscle functional abilities, leading to declines in activities of daily living $[50,51]$. Losses in muscle mass are also associated with declines in resting metabolic rate, which can lead to obesity. In this context, our group has shown increases in resting metabolic rate with ST $[52,53]$. These results from healthy individuals support the hypothesis that ST can reverse or delay the musculoskeletal and metabolic consequences of hormone-dependent cancer treatment. Below is an overview of the evidence from studies that have tested this hypothesis.

3.2. Muscle Mass and Strength. Significant loss of muscle mass is observed within the first year of ADT in PCa patients $[16,17,19,20]$, and the longer the treatment period, the greater the loss in muscle mass [18]. When compared with healthy controls and PCa patients not undergoing ADT, ADT treatment revealed substantial reductions in muscle mass as well as strength $[21,22]$. Ultimately, the loss of muscle mass and strength may be adversely linked to survival because higher levels of muscular strength are associated with lower cancer mortality risk in men, independent of other risk factors [54]. Declines in strength and mass may also occur in BCa patients during treatment, but the cause of this effect is unclear. In one study involving $\mathrm{BCa}$ patients undergoing localized treatment alone versus localized treatment and chemotherapy, there was a slight decline $(<0.5 \mathrm{~kg})$ in fat-free mass over 12 months in the dual treatment group while the localized treatment only group increased fat-free mass [28]. However, this difference was no longer present when results were adjusted for other factors that may have influenced fatfree mass, such as age, race, radiation therapy, baseline body mass index, and baseline fat-free mass. In another study, the effects of chemotherapy and tamoxifen on fat-free mass depended on what method was used to assess fat-free mass [55]. Other investigators have also observed no treatment effect on fat-free mass $[56,57]$ or strength [58] in $\mathrm{BCa}$ patients. Thus, declines in muscle mass and strength appear to be a concern primarily for PCa patients on ADT, given the anabolic role of androgens on muscle mass [59].

In two separate randomized trials, Segal et al. [60, 61] observed strength gains with ST in patients with PCa undergoing ADT. Another study showed that ST improved strength but without changes in whole body fat-free mass in PCa patients on ADT, though a significant increase in quadriceps thickness was reported [62]. Hansen et al. [63] examined the muscle hypertrophic response in PCa patients with and without ADT. Using eccentric resistance exercise, the men not on ADT experienced significant regional hypertrophy whereas the men on ADT did not. No significant differences between groups were observed, but the low statistical power from the small sample size may have contributed to this result. In contrast, we recently detected significant increases 
in thigh muscle volume, directly assessed by computed tomography and whole body fat-free mass with ST in African Americans with PCa on long-term ADT (Hanson et al., unpublished data). BCa patients undergoing treatment are also able to increase their strength levels with ST [41, 57, $58,64]$. However, many of the existing exercise and cancer studies use a mixture of aerobic and ST protocols, which precludes the independent effects of ST. Nevertheless, it is likely that the strength increases reported in these studies are due to ST, given that aerobic exercise training does not typically result in significant strength increases.

3.3. Muscle Power. Muscle power is the product of the force and speed of muscular contractions. It is a strong predictor of the ability to perform the activities of daily living necessary for maintaining a high QoL in older adults [65], to an even greater extent than muscle strength $[66,67]$. For this reason, power has the potential to be an important QoL indicator for patients with cancer. While muscle power declines with advancing age [68], the effects of cancer therapy on muscle power have not yet been reported in PCa or BCa patients.

The authors [42] and others $[45,69]$ have reported significant improvements in peak muscle power with ST in older healthy adults. Since movement speed is unlikely to improve with hormone therapy or decline with ST, it is likely that ST would improve power in cancer patients on hormone therapy, as it does for healthy people, given the reported ST-induced strength increases in these patients. To the best of our knowledge, this has not been studied. However, similar to our unpublished muscle volume data, we observed significant improvements in muscle power at various relative loads with ST (Hanson et al., unpublished data).

3.4. Bone Mineral Density. The hormone therapies used for PCa $[17,22,27]$ and BCa $[15,58,70,71]$ are both associated with a loss in BMD. However, the specific type of $\mathrm{BCa}$ treatment affects BMD differently, as patients on aromatase inhibitors led to declines in BMD while those on tamoxifen experienced significant increases [23-25]. Moreover, bone loss associated with aromatase inhibitor treatment in women with BCa is twofold higher than that of healthy, age-matched postmenopausal women [26]. Chemotherapy treatment for $\mathrm{BCa}$ also reduces $\mathrm{BMD}$, probably as a result of ovarian failure, and occurs in $\sim 71 \%$ of patients $[13,14,58]$. Patients who retained ovarian function did not show any loss in BMD [14]. With the possible exception of fatigue, BMD loss and fracture risk appear to be the single largest consequence of BCa treatment.

We could find only one study each for PCa and BCa reporting the effects of ST on bone mass $[58,62]$. ST for 20 weeks did not result in any changes in BMD in PCa patients [62]. In a randomized clinical trial of BCa patients, six months of home-based aerobic training versus resistance training versus usual care, demonstrated BMD declines of $-0.76 \%,-4.92 \%$, and $-6.23 \%$, respectively, with only a significant difference between the aerobic training and usual care groups [58]. However, the ST program was performed using Thera-Bands and light resistance. Recently, Newton et al. [72] proposed a study that could potentially be the best controlled and most thorough physiological investigation on ST effects in patients on ADT. In particular, they propose to address the impact of ST on bone density as an indicator of fracture risk. They will also determine the effects of impactloading exercise on bone mass. The rationale for this latter aspect of their study is that high and frequent impact forces on long bones are thought to stimulate bone formation.

Despite the lack of direct evidence of significant improvements in BMD in cancer patients, ST can increase BMD in healthy older adults [46, 73-75]. Although not all studies report improvements, those that have compared ST results to controls support the hypothesis that ST is effective for preventing or delaying the loss of BMD over time in older adults. Therefore, it is logical that a similar effect could be present in cancer patients if sufficient intensities are used, despite limited evidence to the contrary [58]. While increases in BMD directly reduce fracture risk, it has been argued that the ST-induced gains $(<5 \%)$ are not sufficient to overcome the estimated $20 \%$ gain in BMD that may be necessary to actually prevent a fracture during falls [76]. Instead, ST may indirectly reduce fracture risk by improving walking mechanics, balance, and strength, some of which have been observed in PCa patients during treatment [62], leading to a reduced risk of falling.

3.5. Fat Mass. Unlike fat-free mass, treatment of both $\mathrm{PCa}$ $[16-20,61,77]$ and BCa $[28,29,55]$ is consistently associated with a significant increase in fat deposition. The increase in fat mass can lead to obesity, diabetes, and metabolic syndrome. Although not directly assessed in previous cancer studies, it is quite likely that PCa and BCa therapies increase fat infiltration in muscle, leading to the accumulation of intermuscular fat [78]. This too has important health implications because elevated levels of intermuscular fat have been linked to insulin resistance and to the development of type 2 diabetes [79]. In addition, fat infiltration is associated with reduced strength [80], poorer leg function [81], and greater incidence of mobility limitations in older adults [82]. While weight gain is widely reported with $\mathrm{BCa}$ treatment, it is not well established if the gain is specifically due to tamoxifen. In a cohort of women who experienced weight gain of $1.7 \mathrm{~kg}$ and increased body fat $2.1 \%$, there were no differences in weight and percent body fat increases between those being treated with tamoxifen and those who were not [29].

Segal et al. [61] found that ST may play a preventative role against the increase in body fat associated with PCa treatment. The patients who performed ST had no change in fat mass while those in the control group experienced significant increases in fat mass. Along these same lines, Galvão et al. [62] reported no change in body fat with ST and ADT, but had no control group for comparison. Similar findings of no significant reductions in body fat have been reported in BCa [57]. Mixed training protocols (ST and aerobic exercise training) can result in a significant loss of fat mass in some [56] but not all [83] studies in BCa patients during treatment. 
3.6. Insulin Resistance. Insulin resistance refers to a reduction in the effectiveness of glucose uptake. This defect is linked to a whole spectrum of disorders, including obesity, cardiovascular disease, and the metabolic syndrome. Basaria et al. [30] observed a substantially higher prevalence of insulin resistance in $\mathrm{PCa}$ patients on ADT than in two other control groups, one in normal healthy controls and the other in a group having a similar disease state, but not on ADT. These results were independent of age and obesity. Similar declines in insulin sensitivity were found after a 12-week prospective study at the onset of ADT [32]. In addition, two independent reviews have corroborated this conclusion showing a strong link between ADT administration and insulin resistance [77, 84] and that this association becomes evident within the first few months of treatment. The authors emphasize the need for men receiving ADT to develop healthy lifestyle practices, such as regular exercise, particularly ST [84]. Furthermore, Basaria [77] found that ADT use for 12 months revealed a higher prevalence of diabetes, metabolic syndrome, and death from cardiovascular disease compared to controls. Similar to PCa patients, BCa patients on hormone therapy also have elevated insulin, glucose, and HbA1c levels [33]. Moreover, low doses of hormone therapy result in a loss of insulin sensitivity in women at a high risk for BCa [31]. Women being treated for $\mathrm{BCa}$ also had higher $\mathrm{C}$-reactive protein levels and incidence rates of metabolic syndrome $[33,85]$.

To the best of our knowledge, however, the independent effect of ST has not been studied in cancer patients undergoing treatment for hormone-dependent cancers. The best evidence available is that BCa patients who underwent supervised ST and home-based aerobic exercise had significant decreases in fasting insulin levels with no change in the control group [83]. There was only a trend for betweengroup differences and no change in fasting glucose levels were found. Patients on ADT undergoing a mixed training protocol had no adjusted group differences after 12 weeks of training for insulin or glucose [86]. In healthy individuals, ST reduces insulin resistance and is as effective as aerobic training in both healthy older adults [48] and diabetics [47]. ST improves the insulin responses during oral glucose tolerance testing and hyperinsulinemic-euglycemic clamps in older men [87, 88] and postmenopausal women [89]. The similarity of findings between ST effects in healthy older adults and those undergoing cancer treatments for other risk factors supports the hypothesis that ST would also produce improvements in insulin resistance in cancer survivors. The data available do not entirely support this, but the independent effects of ST have yet to be evaluated.

3.7. Fatigue and Physical Activity Levels. Fatigue may be the most prevalent and distressing side effect during and after cancer treatment $[4,5]$, as high numbers of patients report suffering from chronic fatigue during this time $[17,34-$ $36,55,90]$. It is likely that both aging and hormone therapy contribute to fatigue by eliciting physiological events (such as anemia, declines in strength and fat-free mass, and increased fat mass) that promote loss in fatigue resistance and the ability or willingness to engage in physical activity. Both $\mathrm{PCa}$ and $\mathrm{BCa}$ patients have reduced physical activity levels after the onset of treatment $[17,91]$. Lower levels of physical activity, in part, result in overall reductions in fitness levels, leading to declines in physical function and ultimately to loss in QoL.

Compliance to regular exercise may break the vicious cycle of fatigue followed by avoidance of physical activity $[4,5]$. Segal et al. $[60,61]$ found that fatigue negatively impacts on activities of daily living and QoL measures but both were improved as a result of ST in men with PCa. They also observed improvements in muscle endurance with the ST group compared to a control group [60]. This finding has been corroborated by others [62], supporting the hypothesis that ST improves fatigue resistance in patients undergoing ADT. Reductions in fatigue with ST have also been observed in patients being treated for $\mathrm{BCa}$ [57], but these reductions were not significantly different from those of a control group. As described for other factors previously, the independent effects of ST are not entirely clear because many studies used both aerobic and ST $[36,92,93]$. There is little doubt, however, that regular exercise reduces fatigue in BCa patients undergoing therapy.

3.8. Physical Function. The loss of physical function during ADT treatment is a common side effect $[18,22,37]$ and is likely the result of decreased muscle mass and physical activity levels coupled with increases in fat mass and fatigue. Investigators who study functional abilities in men with PCa often administer a standardized battery of tests that simulate activities of daily living. This form of testing is seldom reported in women with $\mathrm{BCa}$, rather the declines in function often focus on shoulder dysfunction and lymphedema following surgery rather than hormone treatments [39] or assess function via questionnaire. Reduced shoulder function in $\mathrm{BCa}$ is associated with poorer physical condition, minimal physical activity, increased body mass index, and poorer physical QoL [38], similar to the loss of function and QoL associated with $\mathrm{PCa}$.

ST in men on ADT led to reported improvements of 7 to $27 \%$ in simulated activities of daily living [62]. Others have also reported improved activities of daily living in men with $\mathrm{PCa}$ but have used either combined exercise programs or resistance in which only the lengthening phase of the exercise is loaded (eccentric) $[63,86]$. Studies on the effects of ST on upper body function are limited in $\mathrm{BCa}$ patients, possibly because ST was previously contraindicated due to concerns of lymphedema. However, recent studies have specifically addressed this concern. While no data are available on physical function, adverse events and worsening of lymphedema symptoms did not occur with ST [57, 64, 94]. The fact that women with BCa can safely participate in this type of exercise without adverse consequences provides a rationale for future studies to examine the role of ST on upper body function given the connection between limited function and QoL [38]. 
3.9. Quality of Life. Quality of life is arguably the most egregious side effect resulting from cancer treatment and is intertwined with strength, body composition, fatigue, and physical function. The changes observed with cancer treatment to the above traits are likely to influence QoL. Thus, it is not surprising that men and women undergoing hormone therapy for $\mathrm{PCa}$ and $\mathrm{BCa}$ report reduced QoL indicators $[21,34,36,40,41]$. The questionnaires used to assess QoL varied between studies, although several trends appear to be evident. For example, lower QoL scores were observed for the physical function but not the mental health in both $\mathrm{PCa}$ and $\mathrm{BCa}$ patients [21, 40,41]. Other studies that used QoL questionnaires specific to each cancer type demonstrated reduced overall scores with treatment $[34,36]$. Improved QoL scores with ST are observed in some studies in $\mathrm{PCa}$ and $\mathrm{BCa}$ patient populations $[41,60,61]$, though not in others [57, 63]. Moreover, Ohira et al. [41] reported that several musculoskeletal improvements with ST were correlated with higher QoL indicators.

\section{Conclusions and Future Directions}

Some of the effects of hormone-related treatments for persons with $\mathrm{PCa}$ and $\mathrm{BCa}$ are similar to the typical effects of normal aging but occur at an accelerated pace. These include a long list of musculoskeletal, metabolic, body composition, and functional outcomes. ST appears to have a positive influence on many, but not all of these characteristics. In particular, ST is effective in reversing the loss of strength and function and may be able to slow fat gains and bone losses common to both types of cancer treatments. Due to the widespread use of mixed training protocols, there are some side effects that are reduced from training, but the independent effects of ST cannot be distinguished from effects of other training modalities, such as aerobic exercise. With five-year survival rates for $\mathrm{BCa}$ and $\mathrm{PCa}$ approaching $100 \%$ [2], maintaining high QoL in cancer patients is of paramount importance. In this regard, ST may contribute toward achieving this goal by serving as an effective adjunct therapy and countermeasure to the adverse effects of PCa and BCa treatment.

Over the past decade, the role of exercise has expanded from a means of improving QoL in cancer patients toward addressing specific risk factors resulting from cancer treatment, as well other lifestyle choices that limit function and influence QoL. Future research should address the issue of whether ST can (1) reverse ADT-induced muscle atrophy through direct assessments of muscle area in PCa patients, (2) enhance direct measures of whole body physical function in BCa patients, (3) improve muscle power and bone mineral densities in both populations, and (4) establish a relationship between favorable functional outcomes (activities of daily living, fatigue, QoL) and specific ST-induced adaptations (strength, power, body composition). This additional information will help guide clinicians in exercise prescription designed specifically to reduce comorbidities and particular side effects to enhance QoL in men and women undergoing hormone treatment for $\mathrm{PCa}$ and $\mathrm{BCa}$, respectively.

\section{References}

[1] World Health Organization, "Cancer Facts Sheet," Geneva, Switzerland, September 2010, http://www.who.int/ mediacentre/factsheets/fs297/en/index.html.

[2] A. Jemal, R. Siegel, and E. Ward, "Cancer statistics, 2010," CA Cancer Journal for Clinicians, vol. 60, pp. 277-300, 2010.

[3] R. Yancik, "Cancer burden in the aged: an epidemiologic and demographic overview," Cancer, vol. 80, no. 7, pp. 1273-1283, 1997.

[4] A. Lucía, C. Earnest, and M. Pérez, "Cancer-related fatigue: can exercise physiology assist oncologists?" Lancet Oncology, vol. 4, no. 10, pp. 616-625, 2003.

[5] F. C. Dimeo, "Effects of exercise on cancer-related fatigue," Cancer, vol. 92, no. 6, pp. 1689-1693, 2001.

[6] K. Bylow, S. G. Mohile, W. M. Stadler, and W. Dale, "Does Androgen-deprivation therapy accelerate the development of frailty in older men with prostate cancer? A conceptual review," Cancer, vol. 110, no. 12, pp. 2604-2613, 2007.

[7] B. F. Hurley and S. M. Roth, "Strength training in the elderly: effects on risk factors for age-related diseases," Sports Medicine, vol. 30, no. 4, pp. 249-268, 2000.

[8] A. P. W. Johnston, M. De Lisio, and G. Parise, "Resistance training, sarcopenia, and the mitochondrial theory of aging," Applied Physiology, Nutrition and Metabolism, vol. 33, no. 1, pp. 191-199, 2008.

[9] K. H. Schmitz, A. R. Cappola, C. T. Stricker, C. Sweeney, and S. A. Norman, "The intersection of cancer and aging: establishing the need for breast cancer rehabilitation," Cancer Epidemiology Biomarkers and Prevention, vol. 16, no. 5, pp. 866-872, 2007.

[10] B. F. Hurley, E. D. Hanson, and A. K. Sheaff, "Strength training as a countermeasureto aging muscle and chronic disease," Sports Medicine. In press.

[11] M. B. Williams, J. Hernandez, and I. Thompson, "Luteinizing hormone-releasing hormone agonist effects on skeletal muscle: how hormonal therapy in prostate cancer affects muscular strength," Journal of Urology, vol. 173, no. 4, pp. 1067-1071, 2005.

[12] P. D. Ryan and P. E. Goss, "Adjuvant hormonal therapy in periand postmenopausal breast cancer," Oncologist, vol. 11, no. 7, pp. 718-731, 2006.

[13] T. Saarto, C. Blomqvist, M. Välimäki, P. Mäkelä, S. Sarna, and I. Elomaa, "Chemical castration induced by adjuvant cyciophosphamide, methotrexate, and fluorouracil chemotherapy causes rapid bone loss that is reduced by clodronate: a randomized study in premenopausal breast cancer patients," Journal of Clinical Oncology, vol. 15, no. 4, pp. 1341-1347, 1997.

[14] C. L. Shapiro, J. Manola, and M. Leboff, "Ovarian failure after adjuvant chemotherapy is associated with rapid bone loss in women with early-stage breast cancer," Journal of Clinical Oncology, vol. 19, no. 14, pp. 3306-3311, 2001.

[15] C. K. Osborne, "Tamoxifen in the treatment of breast cancer," New England Journal of Medicine, vol. 339, no. 22, pp. 16091618, 1998.

[16] R. S. Boxer, A. M. Kenny, R. Dowsett, and P. Taxel, "The effect of 6 months of androgen deprivation therapy on muscle and fat mass in older men with localized prostate cancer," Aging Male, vol. 8, no. 3-4, pp. 207-212, 2005.

[17] D. A. Galvão, N. A. Spry, D. R. Taaffe et al., "Changes in muscle, fat and bone mass after 36 weeks of maximal androgen blockade for prostate cancer," BJU International, vol. 102, no. 1 , pp. 44-47, 2008. 
[18] M. E. Levy, S. Perera, G. J. van Londen, J. B. Nelson, C. A. Clay, and S. L. Greenspan, "Physical function changes in prostate cancer patients on androgen deprivation therapy: a 2-year prospective study," Urology, vol. 71, no. 4, pp. 735-739, 2008.

[19] M. R. Smith, "Changes in fat and lean body mass during androgen-deprivation therapy for prostate cancer," Urology, vol. 63, no. 4, pp. 742-745, 2004.

[20] M. R. Smith, J. S. Finkelstein, F. J. McGovern et al., "Changes in body composition during androgen deprivation therapy for prostate cancer," Journal of Clinical Endocrinology and Metabolism, vol. 87, no. 2, pp. 599-603, 2002.

[21] S. Basaria, J. Lieb, A. M. Tang et al., "Long-term effects of androgen deprivation therapy in prostate cancer patients," Clinical Endocrinology, vol. 56, no. 6, pp. 779-786, 2002.

[22] D. A. Galvão, D. R. Taaffe, N. Spry, D. Joseph, D. Turner, and R. U. Newton, "Reduced muscle strength and functional performance in men with prostate cancer undergoing androgen suppression: a comprehensive cross-sectional investigation," Prostate Cancer and Prostatic Diseases, vol. 12, no. 2, pp. 198203, 2009.

[23] R. E. Coleman, L. M. Banks, S. I. Girgis et al., "Skeletal effects of exemestane on bone-mineral density, bone biomarkers, and fracture incidence in postmenopausal women with early breast cancer participating in the Intergroup Exemestane Study (IES): a randomised controlled study," Lancet Oncology, vol. 8, no. 2, pp. 119-127, 2007.

[24] R. Eastell, J. E. Adams, R. E. Coleman et al., "Effect of anastrozole on bone mineral density: 5-year results from the anastrozole, tamoxifen, alone or in combination trial 18233230," Journal of Clinical Oncology, vol. 26, no. 7, pp. 1051-1058, 2008.

[25] R. Eastell, R. A. Hannon, J. Cuzick, M. Dowsett, G. Clack, and J. E. Adams, "Effect of an aromatase inhibitor on BMD and bone turnover markers: 2-Year results of the anastrozole, tamoxifen, alone or in combination (ATAC) trial (18233230)," Journal of Bone and Mineral Research, vol. 21, no. 8, pp. 12151223, 2006.

[26] P. Hadji, J. J. Body, M. S. Aapro et al., "Practical guidance for the management of aromatase inhibitor-associated bone loss," Annals of Oncology, vol. 19, no. 8, pp. 1407-1416, 2008.

[27] M. R. Smith, W. C. Lee, J. Brandman, Q. Wang, M. Botteman, and C. L. Pashos, "Gonadotropin-releasing hormone agonists and fracture risk: a claims-based cohort study of men with nonmetastatic prostate cancer," Journal of Clinical Oncology, vol. 23, no. 31, pp. 7897-7903, 2005.

[28] W. Demark-Wahnefried, B. L. Peterson, E. P. Winer et al., "Changes in weight, body composition, and factors influencing energy balance among premenopausal breast cancer patients receiving adjuvant chemotherapy," Journal of Clinical Oncology, vol. 19, no. 9, pp. 2381-2389, 2001.

[29] M. L. Irwin, A. McTiernan, R. N. Baumgartner et al., "Changes in body fat and weight after a breast cancer diagnosis: influence of demographic, prognostic, and lifestyle factors," Journal of Clinical Oncology, vol. 23, no. 4, pp. 774-782, 2005.

[30] S. Basaria, D. C. Muller, M. A. Carducci, J. Egan, and A. S. Dobs, "Hyperglycemia and insulin resistance in men with prostate carcinoma who receive androgen-deprivation therapy," Cancer, vol. 106, no. 3, pp. 581-588, 2006.

[31] H. Johansson, S. Gandini, A. Guerrieri-Gonzaga et al., "Effect of fenretinide and low-dose tamoxifen on insulin sensitivity in premenopausal women at high risk for breast cancer," Cancer Research, vol. 68, no. 22, pp. 9512-9518, 2008.
[32] M. R. Smith, H. Lee, and D. M. Nathan, "Insulin sensitivity during combined androgen blockade for prostate cancer," Journal of Clinical Endocrinology and Metabolism, vol. 91, no. 4, pp. 1305-1308, 2006.

[33] C. A. Thomson, P. A. Thompson, J. Wright-Bea, E. Nardi, G. R. Frey, and A. Stopeck, "Metabolic syndrome and elevated C-reactive protein in breast cancer survivors on adjuvant hormone therapy," Journal of Women's Health, vol. 18, no. 12, pp. 2041-2047, 2009.

[34] F. Joly, S. M. H. Alibhai, J. Galica et al., "Impact of androgen deprivation therapy on physical and cognitive function, as well as quality of life of patients with nonmetastatic prostate cancer," Journal of Urology, vol. 176, no. 6, pp. 2443-2447, 2006.

[35] A. E. Kyrdalen, A. A. Dahl, E. Hernes, E. Hem, and S. D. Fosså, "Fatigue in prostate cancer survivors treated with definitive radiotherapy and LHRH analogs," Prostate, vol. 70, no. 13, pp. 1480-1489, 2010.

[36] H. M. Milne, K. E. Wallman, S. Gordon, and K. S. Courneya, "Effects of a combined aerobic and resistance exercise program in breast cancer survivors: a randomized controlled trial," Breast Cancer Research and Treatment, vol. 108, no. 2, pp. 279288, 2008.

[37] C. A. Clay, S. Perera, J. M. Wagner, M. E. Miller, J. B. Nelson, and S. L. Greenspan, "Physical function in men with prostate cancer on androgen deprivation therapy," Physical Therapy, vol. 87, no. 10, pp. 1325-1333, 2007.

[38] I. L. Nesvold, S. D. Foss, I. Holm, B. Naume, and A. A. Dahl, "Arm/shoulder problems in breast cancer survivors are associated with reduced health and poorer physical quality of life," Acta Oncologica, vol. 49, no. 3, pp. 347-353, 2010.

[39] J. S. Rietman, P. U. Dijkstra, H. J. Hoekstra et al., "Late morbidity after treatment of breast cancer in relation to daily activities and quality of life: a systematic review," European Journal of Surgical Oncology, vol. 29, no. 3, pp. 229-238, 2003.

[40] K. Dacal, S. M. Sereika, and S. L. Greenspan, "Quality of life in prostate cancer patients taking androgen deprivation therapy," Journal of the American Geriatrics Society, vol. 54, no. 1, pp. 85-90, 2006.

[41] T. Ohira, K. H. Schmitz, R. L. Ahmed, and D. Yee, "Effects of weight training on quality of life in recent breast cancer survivors: the weight training for breast cancer survivors (WTBS) study," Cancer, vol. 106, no. 9, pp. 2076-2083, 2006.

[42] M. J. Delmonico, M. C. Kostek, N. A. Doldo et al., "Effects of moderate-velocity strength training on peak muscle power and movement velocity: do women respond differently than men?" Journal of Applied Physiology, vol. 99, no. 5, pp. 17121718, 2005.

[43] B. F. Hurley, R. A. Redmond, R. E. Pratley, M. S. Treuth, M. A. Rogers, and A. P. Goldberg, "Effects of strength training on muscle hypertrophy and muscle cell disruption in elder men," International Journal of Sports Medicine, vol. 16, no. 6, pp. 378384, 1995.

[44] J. T. Lemmer, D. E. Hurlbut, G. F. Martel et al., "Age and gender responses to strength training and detraining," Medicine and Science in Sports and Exercise, vol. 32, no. 8, pp. 1505-1512, 2000.

[45] R. A. Fielding, N. K. LeBrasseur, A. Cuoco, J. Bean, K. Mizer, and M. A. Fiatarone Singh, "High-velocity resistance training increases skeletal muscle peak power in older women," Journal of the American Geriatrics Society, vol. 50, no. 4, pp. 655-662, 2002. 
[46] A. S. Ryan, F. M. Ivey, D. E. Hurlbut et al., "Regional bone mineral density after resistive training in young and older men and women," Scandinavian Journal of Medicine and Science in Sports, vol. 14, no. 1, pp. 16-23, 2004.

[47] R. J. Sigal, G. P. Kenny, N. G. Boulé et al., "Effects of aerobic training, resistance training, or both on glycemic control in type 2 diabetes: a randomized trial," Annals of Internal Medicine, vol. 147, no. 6, pp. 357-369, 2007.

[48] M. A. Smutok, C. Reece, P. F. Kohhinos et al., "Effects of exercise training modality on glucose tolerance in men with abnormal glucose regulation," International Journal of Sports Medicine, vol. 15, no. 6, pp. 283-289, 1994.

[49] M. S. Treuth, A. S. Ryan, R. E. Pratley et al., "Effects of strength training on total and regional body composition in older men," Journal of Applied Physiology, vol. 77, no. 2, pp. 614620, 1994.

[50] T. R. Henwood and D. R. Taaffe, "Improved physical performance in older adults undertaking a short-term programme of high-velocity resistance training," Gerontology, vol. 51, no. 2, pp. 108-115, 2005.

[51] K. R. Vincent, R. W. Braith, R. A. Feldman et al., "Resistance exercise and physical performance in adults aged 60 to 83," Journal of the American Geriatrics Society, vol. 50, no. 6, pp. 1100-1107, 2002.

[52] E. P. Kirk, J. E. Donnelly, B. K. Smith et al., "Minimal resistance training improves daily energy expenditure and fat oxidation," Medicine and Science in Sports and Exercise, vol. 41, no. 5, pp. 1122-1129, 2009.

[53] J. T. Lemmer, F. M. Ivey, A. S. Ryan et al., "Effect of strength training on resting metabolic rate and physical activity: age and gender comparisons," Medicine and Science in Sports and Exercise, vol. 33, no. 4, pp. 532-541, 2001.

[54] J. R. Ruiz, X. Sui, F. Lobelo et al., "Muscular strength and adiposity as predictors of adulthood cancer mortality in men," Cancer Epidemiology Biomarkers and Prevention, vol. 18, no. 5, pp. 1468-1476, 2009.

[55] R. J. Freedman, N. Aziz, D. Albanes et al., "Weight and Body Composition Changes during and after Adjuvant Chemotherapy in Women with Breast Cancer," Journal of Clinical Endocrinology and Metabolism, vol. 89, no. 5, pp. 2248-2253, 2004.

[56] C. Battaglini, M. Bottaro, C. Dennehy et al., "The effects of an individualized exercise intervention on body composition in breast cancer patients undergoing treatment," Sao Paulo Medical Journal, vol. 125, no. 1, pp. 22-28, 2007.

[57] K. S. Courneya, R. J. Segal, J. R. Mackey et al., "Effects of aerobic and resistance exercise in breast cancer patients receiving adjuvant chemotherapy: a multicenter randomized controlled trial," Journal of Clinical Oncology, vol. 25, no. 28, pp. 4396-4404, 2007.

[58] A. L. Schwartz, K. Winters-Stone, and B. Gallucci, "Exercise effects on bone mineral density in women with breast cancer receiving adjuvant chemotherapy," Oncology Nursing Forum, vol. 34, no. 3, pp. 627-633, 2007.

[59] S. Bhasin, L. Woodhouse, and T. W. Storer, "Proof of the effect of testosterone on skeletal muscle," Journal of Endocrinology, vol. 170, no. 1, pp. 27-38, 2001.

[60] R. J. Segal, R. D. Reid, K. S. Courneya et al., "Resistance exercise in men receiving androgen deprivation therapy for prostate cancer," Journal of Clinical Oncology, vol. 21, no. 9, pp. 1653-1659, 2003.

[61] R. J. Segal, R. D. Reid, K. S. Courneya et al., "Randomized controlled trial of resistance or aerobic exercise in men receiving radiation therapy for prostate cancer," Journal of Clinical Oncology, vol. 27, no. 3, pp. 344-351, 2009.

[62] D. A. Galvão, K. Nosaka, D. R. Taaffe et al., "Resistance training and reduction of treatment side effects in prostate cancer," Medicine and Science in Sports and Exercise, vol. 38, no. 12, pp. 2045-2052, 2006.

[63] P. A. Hansen, C. B. Dechet, C. A. Porucznik, and P. C. LaStayo, "Comparing eccentric resistance exercise in prostate cancer survivors on and off hormone therapy: a pilot study," PM and $R$, vol. 1, no. 11, pp. 1019-1024, 2009.

[64] R. L. Ahmed, W. Thomas, D. Yee, and K. H. Schmitz, "Randomized controlled trial of weight training and lymphedema in breast cancer survivors," Journal of Clinical Oncology, vol. 24, no. 18, pp. 2765-2772, 2006.

[65] S. Herman, D. K. Kiely, S. Leveille, E. O’Neill, S. Cyberey, and J. F. Bean, "Upper and lower limb muscle power relationships in mobility-limited older adults," Journals of Gerontology A, vol. 60, no. 4, pp. 476-480, 2005.

[66] J. F. Bean, D. K. Kiely, S. Herman et al., "The relationship between leg power and physical performance in mobilitylimited older people," Journal of the American Geriatrics Society, vol. 50, no. 3, pp. 461-467, 2002.

[67] J. F. Bean, S. G. Leveille, D. K. Kiely, S. Bandinelli, J. M. Guralnik, and L. Ferrucci, "A comparison of leg power and leg strength within the inCHIANTI study: which influences mobility more?" Journals of Gerontology A, vol. 58, no. 8, pp. 728-733, 2003.

[68] E. J. Metter, R. Conwit, J. Tobin, and J. L. Fozard, "Ageassociated loss of power and strength in the upper extremities in women and men," Journals of Gerontology A, vol. 52, no. 5, pp. B267-B276, 1997.

[69] A. C. Jozsi, W. W. Campbell, L. Joseph, S. L. Davey, and W. J. Evans, "Changes in power with resistance training in older and younger men and women," Journals of Gerontology A, vol. 54, no. 11, pp. M591-M596, 1999.

[70] P. E. Lønning, J. Geisler, L. E. Krag et al., "Effects of exemestane administered for 2 years versus placebo on bone mineral density, bone biomarkers, and plasma lipids in patients with surgically resected early breast cancer," Journal of Clinical Oncology, vol. 23, no. 22, pp. 5126-5137, 2005.

[71] D. M. Reid, J. Doughty, R. Eastell et al., "Guidance for the management of breast cancer treatment-induced bone loss: a consensus position statement from a UK Expert Group," Cancer Treatment Reviews, vol. 34, supplement 1, pp. S3-S18, 2008.

[72] R. U. Newton, D. R. Taaffe, N. Spry et al., "A phase III clinical trial of exercise modalities on treatment side-effects in men receiving therapy for prostate cancer," BMC Cancer, vol. 9, article 210, 2009.

[73] T. Lohman, S. Going, R. Pamenter et al., "Effects of resistance training on regional and total bone mineral density in premenopausal women: a randomized prospective study," Journal of Bone and Mineral Research, vol. 10, no. 7, pp. 10151024, 1995.

[74] A. Menkes, S. Mazel, R. A. Redmond et al., "Strength training increases regional bone mineral density and bone remodeling in middle-aged and older men," Journal of Applied Physiology, vol. 74, no. 5, pp. 2478-2484, 1993.

[75] M. E. Nelson, M. A. Fiatarone, C. M. Morganti, I. Trice, R. A. Greenberg, and W. J. Evans, "Effects of high-intensity strength training on multiple risk factors for osteoporotic fractures: a randomized controlled trial," Journal of the American Medical Association, vol. 272, no. 24, pp. 1909-1914, 1994. 
[76] A. C. Courtney, E. F. Wachtel, E. R. Myers, and W. C. Hayes, "Effects of loading rate on strength of the proximal femur," Calcified Tissue International, vol. 55, no. 1, pp. 53-58, 1994.

[77] S. Basaria, "Androgen deprivation therapy, insulin resistance, and cardiovascular mortality: an inconvenient truth," Journal of Andrology, vol. 29, no. 5, pp. 534-539, 2008.

[78] I. Janssen and R. Ross, "Linking age-related changes in skeletal muscle mass and composition with metabolism and disease," Journal of Nutrition, Health and Aging, vol. 9, no. 6, pp. 408419, 2005.

[79] B. H. Goodpaster, F. L. Thaete, and D. E. Kelley, "Thigh adipose tissue distribution is associated with insulin resistance in obesity and in type 2 diabetes mellitus," American Journal of Clinical Nutrition, vol. 71, no. 4, pp. 885-892, 2000.

[80] B. H. Goodpaster, C. L. Carlson, M. Visser et al., "Attenuation of skeletal muscle and strength in the elderly: the health ABC study," Journal of Applied Physiology, vol. 90, no. 6, pp. $2157-$ $2165,2001$.

[81] M. Visser, S. B. Kritchevsky, B. H. Goodpaster et al., "Leg muscle mass and composition in relation to lower extremity performance in men and women aged 70 to 79: the Health, Aging and Body Composition Study," Journal of the American Geriatrics Society, vol. 50, no. 5, pp. 897-904, 2002.

[82] M. Visser, B. H. Goodpaster, S. B. Kritchevsky et al., "Muscle mass, muscle strength, and muscle fat infiltration as predictors of incident mobility limitations in well-functioning older persons," Journals of Gerontology A, vol. 60, no. 3, pp. 324-333, 2005.

[83] J. A. Ligibel, N. Campbell, A. Partridge et al., "Impact of a mixed strength and endurance exercise intervention on insulin levels in breast cancer survivors," Journal of Clinical Oncology, vol. 26, no. 6, pp. 907-912, 2008.

[84] P. E. Kintzel, S. L. Chase, L. M. Schultz, and T. J. O’Rourke, "Increased risk of metabolic syndrome, diabetes mellitus, and cardiovascular disease in men receiving androgen deprivation therapy for prostate cancer," Pharmacotherapy, vol. 28, no. 12, pp. 1511-1522, 2008.

[85] L. A. Healy, A. M. Ryan, P. Carroll et al., "Metabolic syndrome, central obesity and insulin resistance are associated with adverse pathological features in postmenopausal breast cancer," Clinical Oncology, vol. 22, no. 4, pp. 281-288, 2010.

[86] D. A. Galvão, D. R. Taaffe, N. Spry, D. Joseph, and R. U. Newton, "Combined resistance and aerobic exercise program reverses muscle loss in men undergoing androgen suppression therapy for prostate cancer without bone metastases: a randomized controlled trial," Journal of Clinical Oncology, vol. 28, no. 2, pp. 340-347, 2010.

[87] J. Ibañez, M. Izquierdo, I. Argüelles et al., "Twice-weekly progressive resistance training decreases abdominal fat and improves insulin sensitivity in older men with type 2 diabetes," Diabetes Care, vol. 28, no. 3, pp. 662-667, 2005.

[88] J. P. Miller, R. E. Pratley, A. P. Goldberg et al., "Strength training increases insulin action in healthy 50- to 65-yr-old men," Journal of Applied Physiology, vol. 77, no. 3, pp. 11221127, 1994.

[89] A. S. Ryan, R. E. Pratley, A. P. Goldberg, and D. Elahi, "Resistive training increases insulin action in postmenopausal women," Journals of Gerontology A, vol. 51, no. 5, pp. M199M205, 1996.

[90] N. Tchen, H. G. Juffs, F. P. Downie et al., "Cognitive function, fatigue, and menopausal symptoms in women receiving adjuvant chemotherapy for breast cancer," Journal of Clinical Oncology, vol. 21, no. 22, pp. 4175-4183, 2003.
[91] M. L. Irwin, D. Crumley, A. McTiernan et al., "Physical activity levels before and after a diagnosis of breast carcinoma: the Health, Eating, Activity, and Lifestyle (HEAL) study," Cancer, vol. 97, pp. 1746-1757, 2003.

[92] C. L. Battaglini, A. C. Hackney, R. Garcia, D. Groff, E. Evans, and T. Shea, "The effects of an exercise program in leukemia patients," Sao Paulo Medical Journal, vol. 125, pp. 22-28, 2009.

[93] K. M. Mustian, L. Peppone, T. V. Darling, O. Palesh, C. E. Heckler, and G. R. Morrow, "A 4-week home-based aerobic and resistance exercise program during radiation therapy: a pilot randomized clinical trial," Journal of Supportive Oncology, vol. 7, no. 5, pp. 158-167, 2009.

[94] K. H. Schmitz, R. L. Ahmed, A. Troxel et al., "Weight lifting in women with breast-cancer-related lymphedema," New England Journal of Medicine, vol. 361, no. 7, pp. 664-673, 2009. 


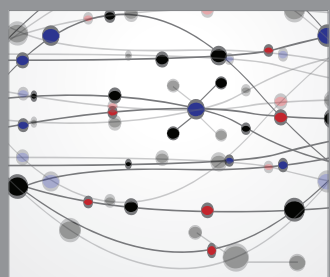

The Scientific World Journal
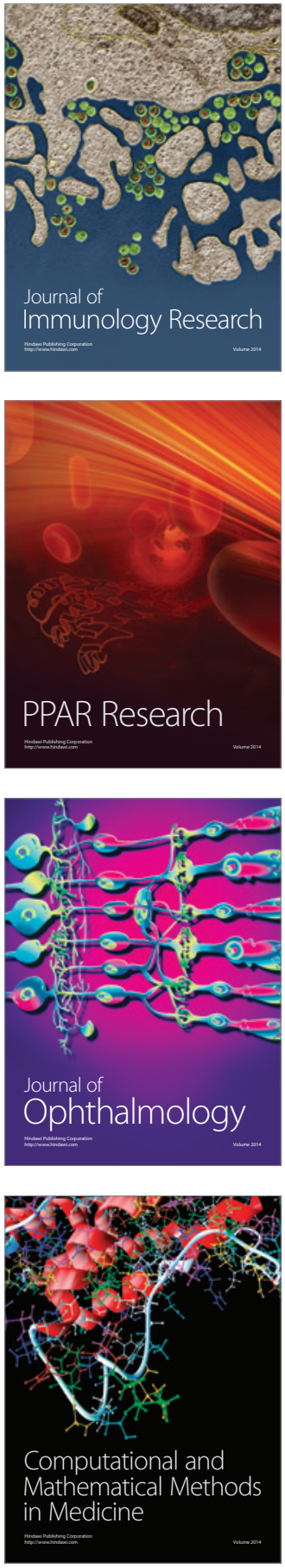

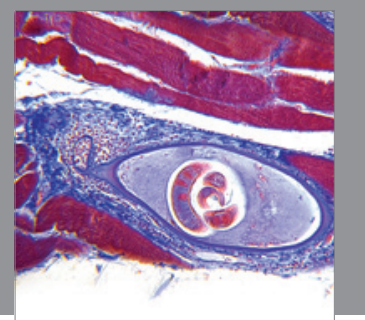

Gastroenterology

Research and Practice
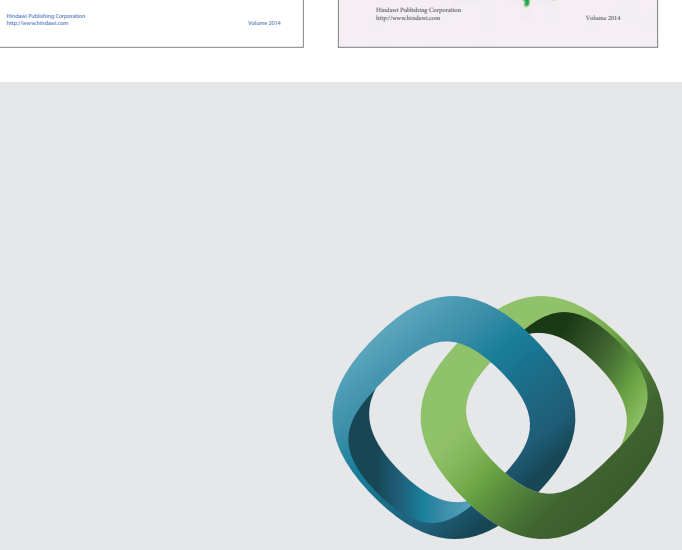

\section{Hindawi}

Submit your manuscripts at

http://www.hindawi.com
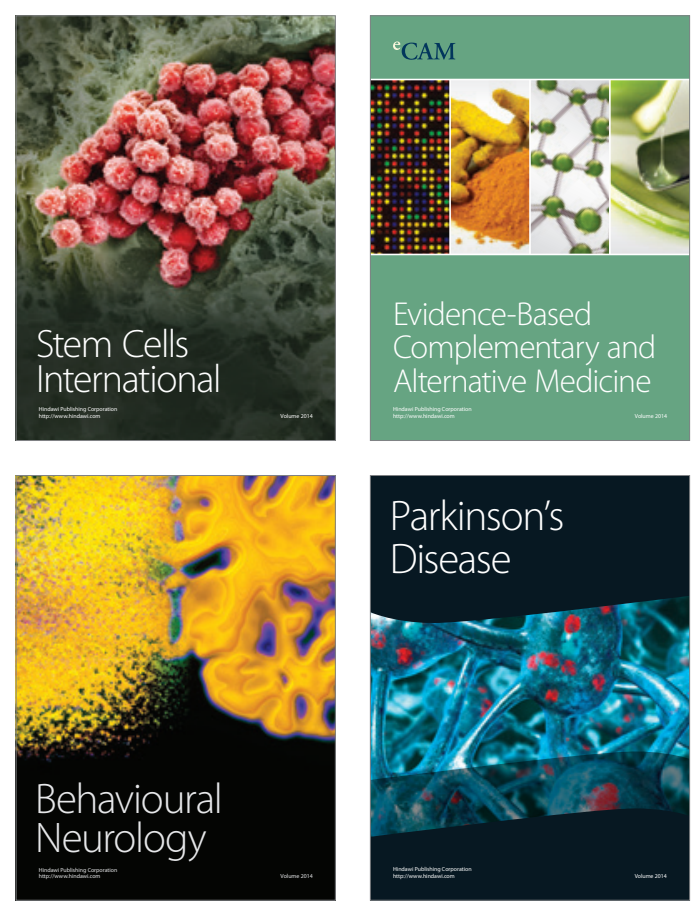

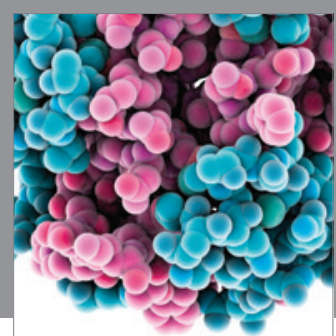

Journal of
Diabetes Research

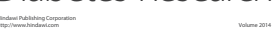

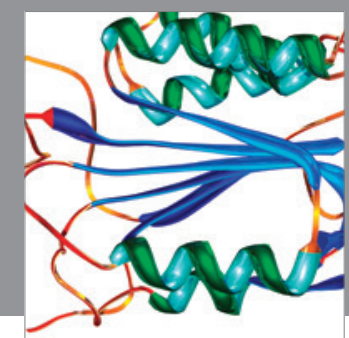

Disease Markers
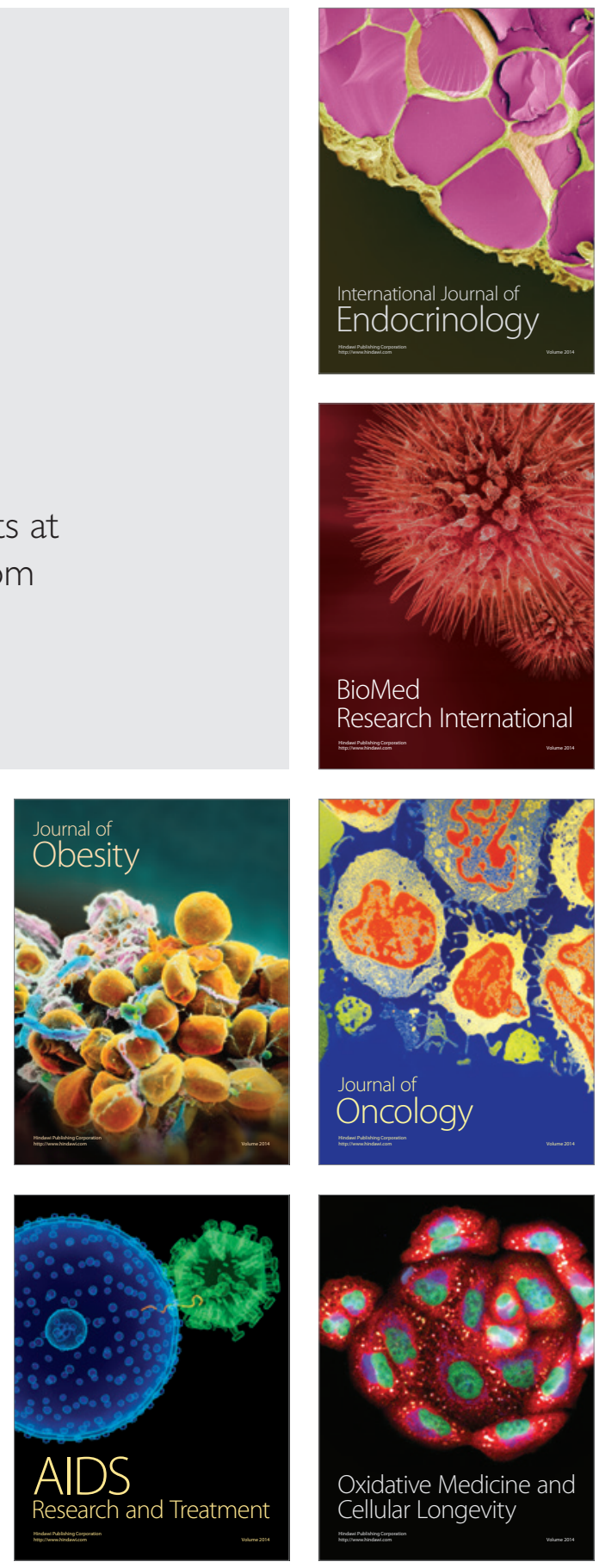